\title{
Glycaemic potency of breakfast predicts cognitive function and mood in adolescent schoolchildren
}

\author{
Renata Micha ${ }^{1}$, Peter J. Rogers ${ }^{2}$ and Michael Nelson ${ }^{1,3}$ \\ ${ }^{1}$ King's College London, London, UK, ${ }^{2}$ University of Bristol, Bristol, UK and ${ }^{3}$ School Food Trust, London, UK
}

The potential influences of nutritional factors on cognitive function (CF) and mood are of major importance, given that adolescence is a crucial period for the development and maturation of both body and mind. Breakfast skipping, as a short-term change in nutritional status, has been shown to have adverse effects on memory and learning ${ }^{(1)}$. Despite the wealth of studies that have been conducted in this area, the macronutrient composition of breakfast that could selectively facilitate $\mathrm{CF}$ after an overnight fast remains unclear ${ }^{(2)}$. It has been suggested that glucose may be mediating the memory-enhancing effects of breakfast, since it is the major source of energy for the central nervous system $^{(3)}$. As pure glucose will be rarely consumed as part of a balanced diet, the interest nowadays is in low-glycaemic index (GI; LGI) meals, and whether they can facilitate performance by minimizing glycaemia fluctuations. The findings from recent studies differ, because of variations in design, especially in relation to the cognitive domains affected. The underlying mechanism remains unclear; it has been recently suggested that perhaps an interaction between glucoregulatory processes, arousal and cortisol could be responsible for any observed effects ${ }^{(4)}$.

The aim of the present study was to investigate the effects of the blood glucose (BG)-raising potential (i.e. glycaemic potency) of breakfast meals on CF and mood in adolescent children. The study is the first of its kind to: take into account both the GI and the glycaemic load (GL), as the latter is a stronger predictor of the glycaemic response; to use breakfast meals that have been tested in relation to their glycaemic and insulinaemic responses; to measure capillary BG and salivary cortisol (SC) levels; to assess mood both as a predictor of $\mathrm{CF}$ and as an outcome of the meals; to report findings in adolescents. Seventy-four schoolchildren (thirty-seven girls and thirty-seven boys) aged 11-14 years were recruited for the study. All participants were habitual breakfast eaters, had normal BMI-for-age and had no known diseases or learning disabilities. Participants were matched and allocated either to the high-GL (HGL) or the low-GL (LGL) group. So, if one participant was allocated to the high-GL group, their match was allocated to the low-GL group. Within each GL group children were given high-GI (HGI) and LGI breakfasts. The children were matched in pairs for gender, form, age ( \pm 6 months), height $( \pm 0.05 \mathrm{~m})$ and BMI centile. The HGL and the LGL meals were not isoenergetic.

\begin{tabular}{lcccc}
\hline & \multicolumn{4}{c}{ Breakfast meals } \\
\cline { 2 - 5 } & HGL-LGI & HGL-HGI & LGL-LGI & LGL-HGI \\
\hline GI meal & 48 & 61 & 48 & 61 \\
GL meal & 41 & 55 & 21 & 28 \\
Energy (kJ) & 1967 & 1962 & 1177 & 1154 \\
Protein (g) & 13.9 & 13.9 & 12.5 & 12.0 \\
Fat $(\mathrm{g})$ & 7.0 & 5.3 & 6.7 & 5.1 \\
Total CHO $(\mathrm{g})$ & 86.7 & 90.4 & 43.2 & 45.2 \\
\hline
\end{tabular}

Repeated measures analysis revealed that the LGI meals were significantly associated with feeling more alert and happy before the tests, while the HGI meals were associated with feeling more tense. Also, the HGL meals were associated with feeling more confident and less sluggish before the tests. There were also significant effects in relation to hunger and thirst; the LGL meals were associated with feeling more hungry and the LGL and HGI meals with feeling more thirsty. Before the CF tests (i.e. 90 min after breakfast) the HGL $(P<0.001)$ and HGI $(P=0.05)$ meals were significantly associated with higher BG levels and, furthermore, the HGI meals $(P=0.032)$ were significantly associated with higher SC levels. Thus, by taking mood, BG and SC levels into account, the following GI associations were revealed: the LGI meals predicted better performance on a word-generation task $(P=0.026)$, and the HGI meals predicted better performance on the Stroop task, but in the HGL group only $(P=0.030)$, a speed of information-processing task $(P=0.014)$ and a serialsevens task $(P=0.025)$. The mechanism that mediates this effect is unknown. The assumption that could be made is that the GI effects are domain specific; an LGI meal, and as a result of that lower BG levels, could result in lower activation of the hypothalamic-pituitaryadrenal (HPA) axis under demanding situations (which is expressed by lower SC levels and feeling less nervous) and therefore better declarative memory performance. On the other hand, an HGI meal, and as a result higher BG levels, could result in stronger activation of the HPA axis under demanding situations (which is expressed by higher SC levels and feeling more nervous) and therefore better vigilance. Overall, the LGI-HGL breakfast appears to be particularly beneficial to school-aged children.

1. Mahoney CR, Taylor HA, Kanarek RB \& Samuel P (2005) Physiol Behav 85, 635-645.

2. Micha R (2008) Glycaemic potency of breakfast and cognitive function in adolescent school children. PhD Thesis, King's College, University of London.

3. Hoyland A, Lawton CL \& Dye L (2008) Neurosci Biobehav Rev 32, 72-85.

4. Gibson EL (2007) Nutr Bull 32, 71-83. 